\title{
Family Meals
}

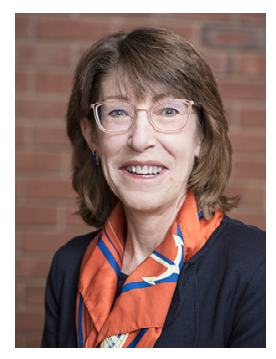

Family meals have changed quite a bit over the past 6 or 8 months. We may be eating at home more with immediate family or eating alone more often because we aren't getting to restaurants. We may be trying more new recipes because of boredom or eating more frozen meals because of fatigue and stress. Looking back over the past year of meal-related articles brought some interesting highlights.

A systematic review published in May concerning the relation between family meal frequency and dietary and family functioning outcomes in children aged 2-18 years concluded that there was some evidence of a positive relationship between family meal frequency and dietary outcomes. ${ }^{1}$ There was stronger evidence for the relation with family functioning outcomes and family meal frequency. These results may be very different when we examine these associations during the pandemic because the family functioning outcomes of family connectedness or cohesion, communication, expressiveness, or conflict or problem-solving might be altered by many stresses we are experiencing.

In a similar vein, family communication was an important consideration in the study by Pratt et al. ${ }^{2}$ In this study, weight talk, dietary habits, and family communication were assessed in 259 patients who had a child aged 2 to 18 years. Patients with higher family communication and younger children were more likely to eat breakfast together. Would this still be true today?

In a cross-sectional analysis of 280 Hispanic seventh and eighth graders with overweight or obesity, mealtime communication was associated with fruit and vegetable consumption in boys and physical activity in girls. ${ }^{3}$ Some results were different for single- vs. dual-headed families. The association between family mealtime communications and fruit and vegetable intake was stronger for girls in single-parent households. For boys, single-parent households did not moderate any of the relationships between mealtime communication and the health outcomes; however, it did have an association with fruit and vegetable intake. The authors conclude that our strategies for behavior change may need to be tailored for family composition. However, in these days of the pandemic, have obesity-related behaviors changed?

Our authors are beginning to submit COVID-19-related work examining some of these questions as well as others. While I hope this will be a blip in our research and practice, I also believe we will learn much about the effects of illness, stress, and food insecurity in our research and practice. I hope we continue to receive such manuscripts.

On a couple of technical notes, reviewers please update your classifications of expertise in Editorial Manager so that we can correctly match manuscripts and reviewers. For authors, to create citations from the papers in JNEB online, I wanted to highlight that you need to need to click on the full-text HTML and then click on "save" for you to get to the export citation function.

Karen Chapman-Novakofski
PhD, RDN
Editor-in-Chief

\section{REFERENCES}

1. Robson SM, McCullough MB, Rex S, Munafò MR, Taylor G. Family meal frequency, diet, and family functioning: a systematic review with metaanalyses. J Nutr Educ Behav. 2020;52: 553-564.

2. Pratt KJ, Skelton JA, Lewis KH, et al. Family meal practices and weight talk between adult weight management and weight loss surgery patients and their children. J Nutr Educ Behav. 2020;52: 579-587.

3. Lebron CN, Agosto Y, Lee TK, et al. Family mealtime communication in single- and dual-headed households among Hispanic adolescents with overweight and obesity. J Nutr Educ Behav. 2020;52: 840-849. 